\title{
Comparison of 2D Models for the Plasma Edge with Experimental Measurements and Assessment of Deficiencies
}

\author{
A.V. Chankin*, D.P.Coster, and the ASDEX Upgrade Team \\ Max-Planck-Institut für Pasmaphysik, Boltzmannstraße 2, 85748 Garching bei München, Germany
}

\begin{abstract}
Systematic discrepancies between 2D fluid edge codes predictions for scrape-off layer and divertor and existing tokamak experiments are described. Possible sources of the discrepancies, including incorrect implementation of the neutral model, the role of fluctuations in the plasma and non-local kinetic effect of parallel heat transport are analysed.
\end{abstract}

JNM keywords: Plasma-Materials Interaction, Plasma Properties

PSI-18 keywords: ASDEX-Upgrade, B2-SOLPS.5.0, Divertor simulation, SOL transport PACS: 52.55.Fa, 52.49.Hf, 52.65.-y, 52.25.Fi

*Corresponding author address: Max-Planck-Institut für Plasmaphysik, Boltzmannstr. 2, 85748 Garching, Germany

*Corresponding author E-mail: Alex.Chankin@ipp.mpg.de

Presenting author: Dr A.V. Chankin

Presenting author E-mail: Alex.Chankin@ipp.mpg.de 


\section{Introduction}

2D edge fluid codes are a powerful tool for predicting scrape-off layer (SOL) and divertor conditions in the next generation of fusion devices including ITER. The main codes in use are: SOLPS (B2-Eirene) [1,2], EDGE2D [3,4] and UEDGE [5]. The codes are believed to be capable of predicting basic trends, being numerically accurate within a factor of 2 (see e.g. refs. [6-8], for SOLPS modelling of L- and H-mode regimes of ASDEX Upgrade).

A number of systematic discrepancies between the code predictions and results of existing experiments were recently identified. This paper describes comparison between experimental results and analytical predictions, and the results of benchmarking the codes against the experiment. A large part of the work was done using the SOLPS code simulating welldiagnosed ASDEX Upgrade (AUG) plasmas, capitalising mainly on high-quality of upstream SOL profiles that can be measured on this machine. The emphasis in the SOLPS modelling was on the outer divertor and outer target plate profiles that receive higher power flux, thus making code predictions more robust. Inner divertor typically receives only $1 / 3$ to $1 / 4$ of the total divertor heat flux (see e.g. [9] for the AUG H-mode plasmas, both experimental and code results), and in the modelling this fraction can vary depending on the assumptions of the degree of ballooning of the perpendicular transport (with a much weaker effect, in percentage terms, on the outer target). Owing to the lower power conducted to the inner divertor, the inner divertor target is usually at least in a partial detachment, bringing in specific difficulties of modelling the detachment process. Therefore, the code solutions for the inner target parameters are very sensitive to the assumptions made in the code leading to large scatter in predictions. 
This paper is organised as follows. Section 2 describes discrepancies between the code predictions and experimental results in the SOL and divertor. In Section 3, possible causes of the discrepancies are discussed. Section 4 is dedicated to one of the possible causes of the discrepancies: non-local kinetic effects in the SOL and divertor. The work is summarised in Section 5 .

The main focus of this work is on high recycling attached or partially detached regimes in magnetic equilibria with poloidal divertor, similar to the one envisaged in ITER. Some persistent difficulties in describing the details of detachment by the $2 \mathrm{D}$ fluid edge codes, mainly in the asymmetries between the two divertors, were reported at very high densities (see e.g. [10] and refs. therein). Such phenomena, occurring when the degree of detachment dramatically increases, are covered in a dedicated paper by M.Wischmeier [11].

\section{Discrepancies in the SOL and divertor}

In the following sub-sections, the three main discrepancies between 2D fluid code predictions and experiment are discussed. For high recycling divertor conditions, with $\mathrm{T}_{\mathrm{e}}$ falling from the midplane towards the divertor target, the SOLPS code was found to underestimate: (a) outer target $T_{e}$ (predicting also flatter $T_{e}$ profiles than in the experiment), leading to higher target $n_{e}$, (b) radial electric field $\mathrm{E}_{\mathrm{r}}$ in the SOL, and (c) parallel ion SOL flows at the low field side (as in [12]; also supported by comparing UEDGE results with JT-60U data [13]). The causality must be: from (a) to (b) via the mechanism of the Debye sheath formation, and then to (c) via the $E_{r}$ influence on the parallel ion flow compensating poloidal $E \times B$ drift. Some other established discrepancies can be regarded as consequences of the mentioned above. For example, divertor $T_{e}$ and $n_{e}$ discrepancies cause radiated power and neutral flux discrepancies. 


\subsection{Parallel ion flow discrepancies}

A systematic study of parallel ion flow in the SOL was performed at JET using the reciprocating Langmuir probe capable of measuring Mach number of the parallel ion flow $\mathrm{M}_{\mathrm{i} \|}$ ('Mach probe'). The probe reciprocated across the SOL at a poloidal location between the outer midplane and the top of the machine. Very large $\mathrm{M}_{\mathrm{i} \|}$, up to 0.6 for the normal toroidal field $\left(\mathrm{B}_{\mathrm{t}}\right)$ direction, by an order of magnitude exceeding those predicted by EDGE2D code simulations, were measured in a series of Ohmic plasmas for normal and reversed $\mathrm{B}_{\mathrm{t}}$ (see results in Fig. 6 of [12]). Part of the disagreement could be attributed to large average flow (averaged between normal and reversed $\mathrm{B}_{\mathrm{t}}$ configurations) caused by the ballooning mechanism of the perpendicular plasma transport that could not be correctly modelled by EDGE2D. Simulation of the ballooning contribution to the parallel ion flow is still problematic for high recycling regimes of tokamaks with poloidal divertors. This issue however is not addressed in the present work, and the reader is referred to refs. $[14,15]$ describing details of experimental results and attempts to model the ballooning component. The difference between $\mathrm{M}_{\mathrm{i} \|}$ for different $\mathrm{B}_{\mathrm{t}}$ configurations, up to $\approx 0.5$, is attributed to drift effects (see below). EDGE2D modelling results for the two Ohmic JET shots analysed earlier in [12], with high and low density, are shown in Fig. 1. Within the first $2 \mathrm{~cm}$ into the SOL, corresponding to the region where the experimental flows peak, the maximum differences between $\mathrm{M}_{\mathrm{i} \|}$ for different $\mathrm{B}_{\mathrm{t}}$ configurations are 0.08 and 0.126 , for high and low Ohmic density plasmas, respectively. There is, therefore, at least a factor of 4 discrepancy between the code and the experiment in this quantity.

A similar discrepancy was established in the SOLPS simulation of the standard Ohmic shot of AUG [14], and also the discrepancy by factor 2 (with the modelling, again, 
underestimating the experimental flow) in UEDGE simulations of L-mode JT-60U plasmas [13]. The ballooning component was absent in the measured flows in AUG and JT-60U where the probe was introduced close to the outer midplane. The same was observed in a series of Ohmic discharges in TCV (see Fig. 2 of [16]). The flows reflected almost symmetrically about zero for all densities, indicating a small contribution from the ballooning mechanism at the outer midplane position.

Large discrepancies between codes and experiments contrast with a reasonably good agreement obtained between experimental $\mathrm{M}_{\mathrm{i} \|}$ measured at the outer midplane and predictions of a simple Pfirsch-Schlüter (P-S) formula for the case of a large aspect ratio tokamak with circular flux surfaces (see e.g. [16-19]), provided all inputs for this formula are taken from experiment. The input parameters are: radial SOL profiles of $n_{e}, T_{e}, T_{i}$ (usually, $T_{i}$ $=T_{e}$ is assumed) and plasma electric potential $V_{p}$. Fig. 2 demonstrates a good agreement with the P-S formula obtained in TCV experiments. It is interesting to note that $\mathrm{M}_{\mathrm{i} \|}$ obtained in SOLPS modelling of AUG Ohmic plasmas were also very close to $\mathrm{M}_{\mathrm{i} \|}$ following from the P-S formula, at the outer midplane position [20]. The discrepancy between experimental and code simulated $\mathrm{M}_{\mathrm{i} \|}$ should therefore be linked to the discrepancy in the input parameters for the P-S formula. Since density and temperature profiles are well matched between the code and experiment, the discrepancy can be attributed to an overestimate of $E_{\mathrm{r}}$ in the code, as will be demonstrated in Section 2.3

In addition to the P-S flow, a 'return parallel flow' fully compensating poloidal $\mathrm{E} \times \mathrm{B}$ drift with the velocity $\mathrm{E}_{\mathrm{r}} / \mathrm{B}_{\theta}$, which is along the P-S velocity at the outer midplane, can emerge in the plasma. This flow was theoretically predicted in [21] and later measured on the TdeV tokamak as a response of parallel ion velocity to the externally applied radial electric field 
[22]. The exact magnitude of this flow, however, is more difficult to predict since, unlike the P-S contribution, it depends on the recycling pattern in the divertor and boundary conditions at the targets. The combined flow velocity which includes both mechanisms: parallel flow fully compensating poloidal $\mathrm{E} \times \mathrm{B}$ drift and the contribution of the ion diamagnetic flow to the parallel P-S flow, for the case of an elliptical plasma and large aspect ratio is given by [14]:

$$
V_{i \| c o m b}=\frac{E_{r}}{B_{\theta}}-2 \frac{a}{R} \frac{B}{B_{\theta}} \frac{\nabla p_{i}}{e n B}\left(1+\frac{\tan ^{2} \alpha}{k^{2}}\right)^{-1 / 2} .
$$

Here $k$ is elongation, and other notations are standard. Eq. (1) provides the maximum estimate for the effects of drifts on the parallel ion SOL flow. At the outer midplane, and for the circular case $(k=1)$, the velocity Eq. (1) is numerically close to that given by the P-S formula, with the same $\nabla p_{i}$ contribution and the $\mathrm{E}_{\mathrm{r}}$ term being larger only by factor $\mathrm{R} / 2 a$, which is typically $\sim 1.5$ for the SOLs of most tokamaks (hence, the P-S velocity can be easily obtained from Eq. (1) by multiplying the $\mathrm{E}_{\mathrm{r}}$ term by $2 a / \mathrm{R} \times \cos \alpha$, remember that $\left.\left(1+\tan ^{2} \alpha\right)^{-1 / 2}=\cos \alpha\right)$. The 'combined' flow, however, may significantly exceed the P-S flow when the position is shifted horizontally from the outer midplance (but still being on the low field side), as in the case of the reciprocating probe position in JET. At the outer midplane, where most of the probe measurements are usually done, given the uncertainties in all input parameters and the fact that the simple P-S formula for a circular high aspect ratio was used, measurements can neither confirm nor rule out the possibility of the 'combined flow' being present in the plasma instead of the P-S flow. Hence, changes in $\mathrm{M}_{\mathrm{i} \|}$ caused by the $\mathrm{B}_{\mathrm{t}}$ direction should more correctly be ascribed to 'drift effects'. 


\subsection{Divertor discrepancies}

The main problem in establishing discrepancies between measurements and code predictions for divertor parameters stems from high sensitivity of code divertor solutions to upstream parameters, with the two most important ones being the input power into the numerical grid and separatrix density. The preference for the separatrix density as one of the control parameters for the code needs to be explained. It is well known that all parameters of the edge plasma are interconnected. In particular, in the parameter scan described in [23] the separatrix density was varied by gas puffing rather than being directly controlled, and the dependence $p^{0.36}$, with $p$ being neutral pressure in the divertor, was obtained for $\mathrm{n}_{\text {sep. }}$. As pointed out in [23], $\mathrm{n}_{\text {sep }}$ is not the parameter that can be directly controlled in the experiment, rather, it is determined by the gas throughput and the effective pumping speed. The latter is affected by the divertor geometry and even by the strike point position on the target. For the purposes of the interpretative simulation described in this paper, however, it is the $\mathrm{n}_{\mathrm{sep}}$, together with the input power into the numerical grid, that can be used for comparison with experimental data.

The $\mathrm{n}_{\text {sep }}$ cannot be determined in present experiments with the required accuracy owing to insufficient accuracy of the equilibrium reconstruction (separatrix position must be determined with the precision of not worse than $2 \mathrm{~mm}$, for AUG conditions) in the presence of sharp $n_{e}$ gradients in the vicinity of the separatrix. Another problem is the necessity to correctly model neutral species, since neutral recycling is crucial in determining divertor conditions. The importance of the neutral model for the divertor solution is evidenced by a large difference in predicted divertor conditions for the standard Ohmic AUG shot depending on whether the fluid neutral model [24] or Monte-Carlo kinetic modelling with Eirene [25] (which predicts colder and denser plasma in the divertor compared to the fluid model) is used in SOLPS. 
The difficulty with pinpointing the separatrix position can be avoided provided good upstream SOL profiles of $n_{e}, T_{e}$ (and ideally, also $T_{i}$ ) are measured with high spatial resolution. The separatrix density can then be determined in the code solution by the requirement that the power flux through the separatrix must match the sum of the power fluxes to the targets plus radiated power in the SOL and divertor. This way of determining the separatrix position makes it dependent on the adopted model for the parallel heat flux to the divertor (Braginskii equations [26], with heat flux limiters, are used in SOLPS). Changing these assumptions will affect the separatrix position in the code. Ideally, the code predicted power to the target should coincide with values obtained from experimental measurements, e.g. from target Lagmuir probes and the infrared camera. For the purposes of determining the separatrix position in the code, however, experimental power deposition is not used directly. Rather, the consistency between direct experimental measurements and power balance calculations is used as one of the criteria for selecting shots ('well-documented shots') suitable for comparison between the code and experiment.

Recent detailed SOLPS modelling of AUG plasmas satisfied the above criteria on both experimental and modelling sides, allowing for the benchmarking of the code against the experimental data. At medium to high densities, the code was found to overestimate divertor $\mathrm{n}_{\mathrm{e}}$ and underestimate $\mathrm{T}_{\mathrm{e}}$ (see [9] for H-mode and Fig. 3 for an Ohmic AUG shot). SOLPS simulations also predict flatter $\mathrm{T}_{\mathrm{e}}$ profiles than in the experiment, as seen in Fig. 3. A higher recycling level in the code reflects larger target flux that scales roughly as $n \sqrt{T}$. For a given plasma pressure $p=n T$ which tends to be conserved along the field lines for as long as the plasma is not too detached, this implies the scaling $p / \sqrt{T}$ for the recycling, in agreement with the code predicting lower $T_{e}$. Owing to the presence of supra-thermal ions and electrons 
in the divertor (see Section 4), by 'temperature' $T$ one understands here its measure in a broader sense, as $2 / 3$ of the energy averaged over the distribution function: $2 / 3\left\langle E_{e, i}\right\rangle$.

At the same time, in low density AUG Ohmic shots (close to the lowest density obtainable), good agreement between SOLPS code predictions and target Langmuir probe data, similar to earlier observations from TCV, was obtained (see [10] and refs. therein).

\subsection{Scrape-off layer $E_{\mathrm{r}}$ discrepancy}

As was pointed out in Section 2.1, both experimentally measured and code predicted values of $\mathrm{M}_{\mathrm{i}}$ near the outer midplane position agree with the simple P-S formula, but the code obtained $\mathrm{M}_{\mathrm{i}}$ are much smaller than the experimental values. With the SOLPS solutions well matching upstream SOL profiles of $n_{e}, T_{e}$ (and $T_{i}$, with a somewhat poorer quality owing to inferior experimental profiles), the only possibility to explain the discrepancy between experimental and code predicted changes of $\mathrm{M}_{i||}$ related to the $\mathrm{B}_{\mathrm{t}}$ reversal is to assume that the value of $E_{\mathrm{r}}$ is also underestimated in the codes, as was first suggested in [20]. This was later confirmed for Ohmic shots of AUG, using Langmuir probe and Doppler reflectometer data to determine experimental $\mathrm{E}_{\mathrm{r}}$ [27]. The experimental $-\mathrm{eE}_{\mathrm{r}} / \nabla \mathrm{T}_{\mathrm{e}}$ ratio (with both quantities taken at the outer midplane) was found to be $\approx 3.1$, with the SOLPS predicted value close to zero. Similarly large disagreement follows from the comparison between $-\mathrm{eE}_{\mathrm{r}} / \nabla \mathrm{T}_{\mathrm{e}}$ ratios in $\mathrm{JET}$ experiments, $\approx 1.6$ on average, and low values of these ratios in the EDGE2D code solutions, not exceeding 0.5 (see Fig. 1). In addition, experimental $-\mathrm{eE}_{\mathrm{r}} / \nabla \mathrm{T}_{\mathrm{e}}$ ratios $\approx 2.5$ were obtained in JT-60U, and from 3 to 5 - in TCV [27]. These experimental results were obtained from reciprocating Langmuir probes, using formula $\mathrm{e}\left(\mathrm{V}_{\mathrm{p}}-\mathrm{V}_{\mathrm{f}}\right) / \mathrm{T}_{\mathrm{e}}=3$ for deriving plasma potential $\mathrm{V}_{\mathrm{p}}$ from measured $\mathrm{T}_{\mathrm{e}}$ and floating potential $\mathrm{V}_{f}$. 
The $\mathrm{E}_{\mathrm{r}}$ discrepancy between the codes and experiments provides a link between the two other discrepancies described in the previous sections, as pointed out in Section 2. All three discrepancies therefore appear to be linked together and are expected to have a common origin. Possible causes of these discrepancies are discussed next.

\section{Possible causes of the discrepancies}

Three possible explanations for the discrepancies have been proposed so far, based on the features in the codes that are either missing or being suspected for incorrect implementation of physics mechanisms. They are: possible drawbacks of neutrals treatment in Eirene, possible influence of plasma fluctuations of time-averaged parameters (codes ignore plasma fluctuations and assume quasi-steady-state evolution of parameters), and kinetic effects of parallel plasma transport, poorly accounted for in fluid codes.

The primary discrepancy must be the one in the divertor parameters, with the two others (in the $E_{r}$ and $V_{i \|}$ in the SOL) being consequences. The original suspicion fell on possible deficiencies in Eirene that can lead do increased recycling levels in the divertor by excessive ionization of neutrals due to their restricted mobility, attributed to either their unrealistically large ionization rates or to small velocity. Extensive sensitivity studies of SOLPS solutions to neutral physics implemented in Eirene and described in [9,25] were carried out. They included variation of neutral atomic and molecular reaction rates, transport coefficients and power sharing between ion and electron channels, and even artificial increase in the wall temperature by factor 10 to speed up molecules. None of the measures however bridged the gap between the experiment and code results. It still cannot be completely ruled out that neutrals are responsible for discrepancies in the divertor (for example, due to the influence of 
hydrocarbons that are presently not included in SOLPS), and sensitivity studies will be continued.

The second explanation, involving the role of fluctuations in influencing time-averaged secondary parameters (which are products of primary parameters such as $n_{e}, T_{e}$, raised to various powers) $[28,29]$, has not yet been formulated in any detail. The present $2 \mathrm{D}$ fluid codes implicitly assume, for example, that the time-averaged parallel electron heat conduction to the target scales as $\left(\bar{T}_{e}\right)^{7 / 2}$ (according to Braginskii equations [26]), where $T_{e}$ is upstream electron temperature and $(\ldots)$ denotes time-averaging, and assuming upstream $\mathrm{T}_{\mathrm{e}}$ $>>$ than that at the target). The correct time-averaging procedure, in contrast, should use $T_{e}^{7 / 2}$, yielding $\overline{T_{e}^{7 / 2}}$. The potentials of this explanation for eliminating discrepancies in the divertor will be examined in the future, using both upstream Langmuir probe fluctuation measurements and turbulence code predictions (in particular, analysis of the ESEL code predictions for parallel electron heat flux fluctuations in JET plasmas, the work currently underway). The third explanation is discussed next.

\section{Kinetic effects in the SOL and divertor}

The importance of kinetic effects in the parallel plasma transport comes from strong dependence of the charged particle Coulomb collision time on its velocity, $\sim \mathrm{v}^{3}$, leading to the $v^{4}$ scaling for the collisional mean free path. As a result, even when the bulk, thermal plasma is strongly collisional, with $\lambda_{\text {coll }} / \mathrm{L}_{\|}<<1$, supra-thermal particles may only be weakly collisional, or even almost collisionless. One important consequence of this is for the formation of the Debye sheath near the material surface. The sheath is formed by a small fraction of electrons from the tail of the distribution function capable of overcoming the 
potential barrier in front of the solid surface (typically $\sim 3 \mathrm{~T}_{\mathrm{e}} / \mathrm{e}$, for a near-Maxwellian distribution, deuterium plasma and $\mathrm{T}_{\mathrm{e}} \approx \mathrm{T}_{\mathrm{i}}$ ). Such electrons are much less collisional than the bulk of the electron component. As was demonstrated using simple kinetic estimates in [30], for the $T_{e}$ falling towards the target (the usual situation) the sheath will be formed not by local, near target, electrons, but by those originating upstream of the target at a distance of $\lambda_{\text {coll }}\left(T_{e, \text { upstream }}\right)$ characteristic of a higher, upstream $T_{e}$. This should increase the Debye sheath drop and also modify current-voltage Langmuir probe characteristics leading to an overestimate of the $T_{e}$ by probes. Similar results were obtained by the comparison between SOLPS modelled $\mathrm{T}_{\mathrm{e}}$ profiles near the target of TCV and a simple kinetic model extending that of Wesson [31], and confirmed by kinetic Fokker-Planck modelling that also predicts a large overestimate of $T_{e}$ by divertor Langmuir probes for typical plasma conditions in DIII-D and TdeV [32]. In contrast, good agreement between divertor Thomson scattering and target plate probe $T_{e}$ was obtained at typical densities and power levels for the DIII-D SOL plasma, consistent with the kinetic code predicting the fraction of non-thermal electrons near the target plate being less than $1 \%$ [33].

The consequences of non-local kinetic transport of supra-thermal electrons can be even stronger for the parallel electron heat conduction, which, owing to $\chi_{\mathrm{e} \|}>>\chi_{\mathrm{i} \|}$, is more important than the ion heat conduction. It is well known (see e.g. [34]) that supra-thermal electrons with velocities in the range 3-5 of electron thermal velocity $\mathrm{v}_{T_{e}}=\sqrt{T_{e} / m_{e}}$ contribute the bulk of the parallel electron heat flux. The peak of the heat flux density $\mathrm{v}_{\|} \mathrm{v}^{2} f_{e}$ is reached at $\mathrm{v}_{e} \approx 3.7 \mathrm{v}_{T_{e}}$ (see Fig. 4), corresponding to kinetic energy of $\approx 6.8 \mathrm{~T}_{\mathrm{e}}$. 
Kinetic Fokker-Planck modelling of parallel electron heat flux shows that the deviation from the classical collisional Braginskii (or Spitzer-Härm) formulas for $\chi_{\mathrm{e} \|}$ starts already at $\lambda_{\text {coll }} / \mathrm{L}_{\|}$ $>0.01$, and due to high mean free paths of supra-thermal electrons the heat transport acquires a non-local character. The impact of kinetic effects on the classical expression for $\chi_{\mathrm{e} \|}$ is twofold: the depletion of supra-thermal population reduces $\chi_{\mathrm{e} \|}$ in the hotter upstream plasma, while near the target the plasma becomes over-populated by high energy tail electrons originating from the upstream, steep $T_{e}$ gradient region (see, e.g. [35-37,32]). This is illlustrated in Fig. 5, which shows electron energy flux density distributions from the FokkerPlanck simulation of a typical divertor case with $\lambda_{\text {coll }} / L_{\|}=0.1$ upstream and a large $T_{e}$ drop to the target [32]. Both the depletion of electron population at the 'hot' end (upstream) and overpopulation by high energy electrons stretching beyond $\mathrm{E} / \mathrm{T}_{\mathrm{e}}=10$ at the 'cold' end near the target, compared to the Braginskii's result, can be seen.

As a way of avoiding complexity of Fokker-Planck calculations, an alternative method was offered in [38] using a delocalisation formula for $\chi_{\mathrm{e} \|}$. The results were successfully tested against the direct Fokker-Planck modelling for laser-produced plasmas. The delocalisation formula describes the local $\chi_{\mathrm{e} \|}$ as a spatial (along field lines) integral of the product of the Spitzer-Härm (SH) heat conductivity and a kernel ('convolution kernel'). It could correctly describe both the reduction of the heat flux, compared to the $\mathrm{SH}$ value, in the steep $\mathrm{T}_{\mathrm{e}}$ front occurring in laser-irradiated targets (resulting in the 'flux limit' for the parallel heat flux of 0.1 of the free-streaming flux [38]) and its enhancement at the base of the heat front due to nearly collisionless electrons streaming away from the top of the heat front (the so called 'preheating'). A modified Luciani's formula using the truncation of the integral at the entrance to the magnetic pre-sheath was used for modelling ASDEX plasmas [39]. Delocalisation formulas assume some sort of linearity (albeit, focusing more on the nearby 
locations) in the contributions from remote places to the local electron distribution function, and their results should be more robust when the deviations from the Maxwellian are small. Their predictive capability is also limited by a rather arbitrary choice of a convolution kernel, with a large range of kernel reported in the literature reflecting the variety of plasma conditions in particular experiments, with some formulas tested under certain particular conditions showing unsatisfactory performance under different conditions [40]. They can't therefore serve as a replacement for full kinetic Fokker-Planck simulations which, in turn, predict a rather large scatter of deviations from the classical expressions for $\chi_{\mathrm{e}}$ depending mainly on experimental conditions: flux limits in the range $0.03-0.8$ were reported in the literature [41]. The importance of full Fokker-Planck modelling was confirmed by kinetic ASCOT code modelling (using test electrons launched against the plasma background supplied by the SOLPS solution) of an AUG H-mode shot, where energy composition of kinetic electron losses to the divertor target was found to be very sensitive to the distribution of the background electron density and temperature along the field lines, which in turn are sensitive to the details of magnetic geometry and ionization and radiation patterns in the divertor region [42].

Owing to the sensitivity of kinetic code results to details of experimental conditions, future development of edge codes should be aimed at combining Fokker-Planck calculations with today's main 2D edge fluid codes: SOLPS, EDGE2D and UEDGE. The latter incorporate the necessary level of detail in physical processes, including effects of real magnetic geometry, neutrals and impurities behaviour and anomalous perpendicular transport (without, however, including turbulence itself). Regarding the inclusion of kinetic effects, the present $2 \mathrm{D}$ edge fluid codes share the philosophy expressed in [43] that parallel energy transport is determined by classical conduction and convection, with kinetic corrections to heat diffusivities $\chi_{\| \text {i,e }}$ at 
low (separatrix) collisionalities $v_{i, e}^{*}$. The codes use ad-hoc constant flux limiters for parallel heat and viscous fluxes (e.g., the heat flux limit of 0.3 is used in SOLPS for modelling ITER [2]) and ignore the enhancement of the electron heat conduction in the divertor. Large scatter in predicted flux limiters for $\chi_{\mathrm{e}}$ and a possibility of large enhancement factors for $\chi_{\mathrm{e} \|}$ at sufficiently low $\mathrm{T}_{\mathrm{e}}$ can render classical collisional formulas inapplicable for SOL and divertor modelling in some conditions. A proper Fokker-Planck kinetic treatment of parallel heat transport can be the only alternative.

The importance of non-local kinetic effects is not only restricted to transport phenomena. They can also strongly modify atomic physics rates, especially in low temperature, detached plasma conditions $[32,44]$.

\section{Summary}

Accumulating evidence suggests that solutions of the main 2D edge fluid codes (SOLPS, EDGE2D and UEDGE) are at partial, but systematic, variance with some experimentally measured parameters in the SOL and divertor. In the SOL, the codes systematically underestimate the extent to which the reversal of the toroidal field $\left(\mathrm{B}_{\mathrm{t}}\right)$ in the experiment changes the parallel ion flow velocity. In addition, the codes underestimate radial electric field $\mathrm{E}_{\mathrm{r}}$ in the SOL.

Code predictions for divertor conditions critically depend on the knowledge of radial profiles in the SOL, and especially, on the plasma separatrix density $\mathrm{n}_{\text {sep }}$. The latter, together with the input power into the numerical grid, is one of the two key parameters determining the divertor solution. The value of $\mathrm{n}_{\text {sep }}$ is however poorly defined in the experiment due to insufficient accuracy of the equilibrium reconstruction. Recent interpretive edge modelling 
using SOLPS (B2-Eirene) code package capitalised on excellent diagnostic capabilities of AUG, mainly in providing well resolved upstream radial $n_{e}$ and $T_{e, i}$ profiles. In conjunction with the global power balance and experimental profiles in the divertor, this made the modelling an over-constrained problem, eliminated uncertainty in the $\mathrm{n}_{\text {sep }}$ and allowing code benchmarking. For high recycling divertor conditions, the code was found to predict colder (lower $T_{e}$ ) and denser (higher $n_{e}$ ) plasmas in the divertor compared to experiment. Divertor discrepancies are consistent with those established in the SOL: lower target $\mathrm{T}_{\mathrm{e}}$ predicted by the codes, via the mechanism of the Debye sheath formation, reduces $E_{r}$ in the SOL, which in turn reduces the contribution of the $\mathrm{E} \times \mathrm{B}$ drift to the generation of parallel ion flow.

A number of possible causes for the observed discrepancies were considered. They include physics processes and effects that are either missing in the codes or may be described incorrectly: (a) deficiencies of the neutral model, which is not detailed enough in the inclusion of all possible reactions, (b) role of plasma fluctuations (not included in the codes; the codes assume quasi-steady-state parameters), and (c) non-local kinetic effects in the parallel plasma transport (poorly accounted for in the codes). The absence of non-local kinetic effects can be a critical drawback of the $2 \mathrm{D}$ edge fluid codes in their application to tokamak plasmas that are known to be insufficiently collisional. Both simple estimates and kinetic code results indicate that missing kinetic effects can be very important in the parallel heat flux. In the present day edge fluid codes, kinetic effects are treated in a simplistic way via the use of constant flux limiters. This however neglects the surplus of supra-thermal particles in the cold divertor plasma and their effect on heat conduction (the so called 'preheating') and on an increase in the Debye sheath drop at the target(s). Some of the SOLPS and EDGE2D cases had similar separatrix electron collisionality as that envisaged in 
the reference ITER H-mode scenario. The results of the benchmarking may therefore have wide implications for future edge modelling activity. 


\section{References}

[1] Schneider R, Reiter D, Zehrfeld H P, Braams B, Baelmans M, et al., J. Nucl. Mater. 196198 (1992) 810.

[2] Reiter D, J. Nucl. Mater. 196-198 (1992) 80.

[3] Taroni A, Corrigan G, Radford H, Simonini R, Spence J, et al., Contrib. Plasma Phys. 32 (1992) 438.

[4] Simonini R, Corrigan G, Radford G, Spence J, Taroni A, Contrib. Plasma Phys. 34 (1994) 368.

[5] Rognlien T, Brown P, Campbell R, Kaiser T, Knoll D, et al., Contrib. Plasma Phys. 34 (1994) 362, Fourth Workshop on Plasma Edge Theory, October 1993, Varenna, Italy.

[6] Schneider R, Coster D P, Kallenbach A, Borrass K, Bosch H S, et al., 'Test of the Predictive Capability of B2-EIRENE on ASDEX Upgrade', in: $17^{\text {th }}$ International Conf. on Plasma Physics and Controlled Fusion Research, Yokohama, Japan, 19-24 October (1998), paper F1-CN-69/THP2/05.

[7] Schneider R, Coster D P, Neuhauser J, Bosch H S, Fuchs J C, et al., 'B2-Eirene modelling of the CDH mode in ASDEX Upgrade', and http://www.rzg.mpg.de/ dpc/rfs_poster_eps95/paper/eps95/eps95.html.

[8] Coster D P, Bonnin X, Borrass K, Bosch H-S, Braams B, et al., in Proc. of $18^{\text {th }}$ IAEA Conference, Fusion Energy, Sorrento, Italy, October 2000, (CD-ROM), paper IAEA-CN77/EXP5/32, Vienna, 2000, IAEA.

[9] Chankin A V, Coster D P, Dux R, et al., Plasma Phys. Control. Fusion 48 (2006) 1839.

[10] Wischmeier M, Bonnin X, Coster D, Chankin A V, Kallenbach A, Müller H W, and the ASDEX Upgrade Team, to be published in Contrib. Plasma Phys. 48 (2008).

[11] Wischmeier $\mathrm{M}$ et al., these proceedings, paper O-25. 
[12] Erents S K, Pitts R A, Fundamenski W, Gunn J P and Matthews G F, Plasma Phys. Control. Fusion 46 (2004) 1757.

[13] Asakura N, Takenaga H, Sakurai S, et al., Nucl. Fusion 44 (2004) 503.

[14] Chankin A V, Coster D P, Asakura A, et al., Nucl. Fusion 47 (2007) 762.

[15] Fundamenski W, Garcia O E, Naulin V, et al., Nucl. Fusion 47 (2007) 417.

[16] Pitts R A, Horacek J, and the TCV Team, proc. of $34^{\text {th }}$ EPS Conference on Plasma Phys. Warsaw, 2 - 6 July 2007 ECA Vol.31F, O4.007 (2007).

[17] Asakura N, Sakurai S, Shimada M, Koide Y, Hosogane N, and Itami K, Phys. Rev. Lett. $84(2000) 3093$.

[18] Asakura N, ITPA and Divertor Topical Group, J. Nucl. Mater. 363-365 (2007) 41.

[19] Pitts R A, Horacek J, Fundamenski W, et al., J. Nucl. Mater. 363-365 (2007) 505.

[20] Chankin A V, Coster D P, Dux R, et al, 'Critical issues identified by the comparison between experimental and SOLPS modelling on ASDEX Upgrade', in Fusion Energy 2006 (Proc. 21 st Int. Conf. Chengdu, 2006) (Vienna: IAEA) CD-ROM file TH/P6-15 and http://www-naweb.iaea.org/napc/physics/FEC/FEC2006/html/index.htm.

[21] Tendler M and Rozhansky V, Comments Plasma Phys. Control. Fusion 13 (1990) 191.

[22] MacLatchy C S, Boucher C, Poirier D A, Gunn J P, Stansfield B L, Zuzak W W, J. Nucl. Mater. 196-198 (1992) 248.

[23] Kukushkin A S, Pacher H D, Pacher G W, Janeschitz G, Coster D, Loarte A and Reiter D, Nucl. Fusion 43 (2003) 716.

[24] Coster D P, Chankin A V, Conway G D, et al., proc. of $32^{\text {nd }}$ EPS Conference on Plasma Phys. Tarragona, 27 June - 1 July 2005 ECA Vol.29C, P1.008 (2005).

[25] Chankin A V, Coster D P, Dux R, et al., J. Nucl. Mater. 363-365 (2007) 335.

[26] Braginskii S I, Review of Plasma Physics (Consultants Bureau, New York, 1965), Vol. 1, p. 205. 
[27] Chankin A V, Coster D P, Asakura A, et al., Nucl. Fusion 47 (2007) 479.

[28] Fundamenski W, private communication (2006).

[29] Krasheninnikov S I, private communication (2007).

[30] Wesson J A, Plasma Phys. Control. Fusion 37 (1995) 1459.

[31] Horacek J, Pitts R A, Stangeby P C, Batishchev O, Loarte A, J. Nucl. Mater 313-316 (2003) 931.

[32] Batishchev O V, Krasheninnikov S I, Catto Peter J, et al., Phys. Plasmas 4 (1997) 1672.

[33] Watkins J G, Batishchev O, Boedo J, et al. J. Nucl. Mater. 266-269 (1999) 980.

[34] Stangeby P C, 2000 The Boundary of Magnetic Fusion Devices (Bristol: IOP Publishing)

[35] Chodura R, Contrib. Plasma Phys. 28 (1988) 4/5, 303.

[36] Kukushkin A S, Runov A M, Contrib. Plasma Phys. 34 (1994) 2/3, 204.

[37] Kupfer K, Harvey R W, Sauter O., Schaffer M J, Staebler G M, Phys. Plasmas 3 (1996) 3544.

[38] Luciani J F, Mora P, Virmont J, Phys. Rev. Lett. 51 (1983) 1664.

[39] Lackner K, Ditte U, Fussmann G, et al., Plasma Phys. and Control. Nucl. Fusion Research, $10^{\text {th }}$ Conf. Proceedings, London, 12-10 Sep. 1984, Nucl. Fusion Supplement (1985) Vol. 1, p.319, IAEA-CN-44/A-V-4.

[40] Alouani Bibi F, Matte J-P, Phys. Rev. E 66 (2002) 066414.

[41] Fundamenski W, Plasma Phys. Control. Fusion 47 (2005) R163.

[42] Aho-Mantila L K, Kurki-Suonio T, Chankin A V, Coster D P, Sipilä S K, Plasma Physics and Controlled Fusion 50 (2008) 065021.

[43] Loarte A, Lipschultz B, Kukushkin A S, et al., Progress in the ITER Physics Basis.

Chapter 4: Power and particle control, Nucl. Fusion 47 (2007) S203-S263.

[44] Allais F, Matte J P, Alouani-Bibi, Kim C G, Stotler D P, Rognline T D, J. Nucl. Mater, 337-339 (2005) 246. 


\section{Figure captions}

Fig. 1. Mach numbers of the parallel ion flow at the reciprocating probe position, $T_{e}$ and plasma potentials at outer midplane, for high (a) and low (b) density Ohmic JET plasmas simulated by EDGE2D. The distance from the separatrix is mapped to the outer midplane position (replicated from [14], with minor alterations).

Fig. 2. Comparison of measured and calculated Mach numbers in the SOL of TCV for upper (SNU) and lower (SNL) single-null configurations. The field independent (ballooning) contribution was offset from SNL data (replicated from [16], with minor alterations).

Fig. 3. Simulated (with SOLPS code) and measured (with target Langmuir probes) $n_{e}$ and $T_{e}$ profiles along outer target for standard Ohmic AUG shot (replicated from [25], with minor alterations).

Fig. 4. Electron parallel energy flux density for strongly collisional plasmas vs. dimensionalised electron velocity (replicated from [33], p.658).

Fig. 5. Electron energy flux density vs. electron energy normalized to local $T_{e}$ (replicated from [32]), see text for the legend. 

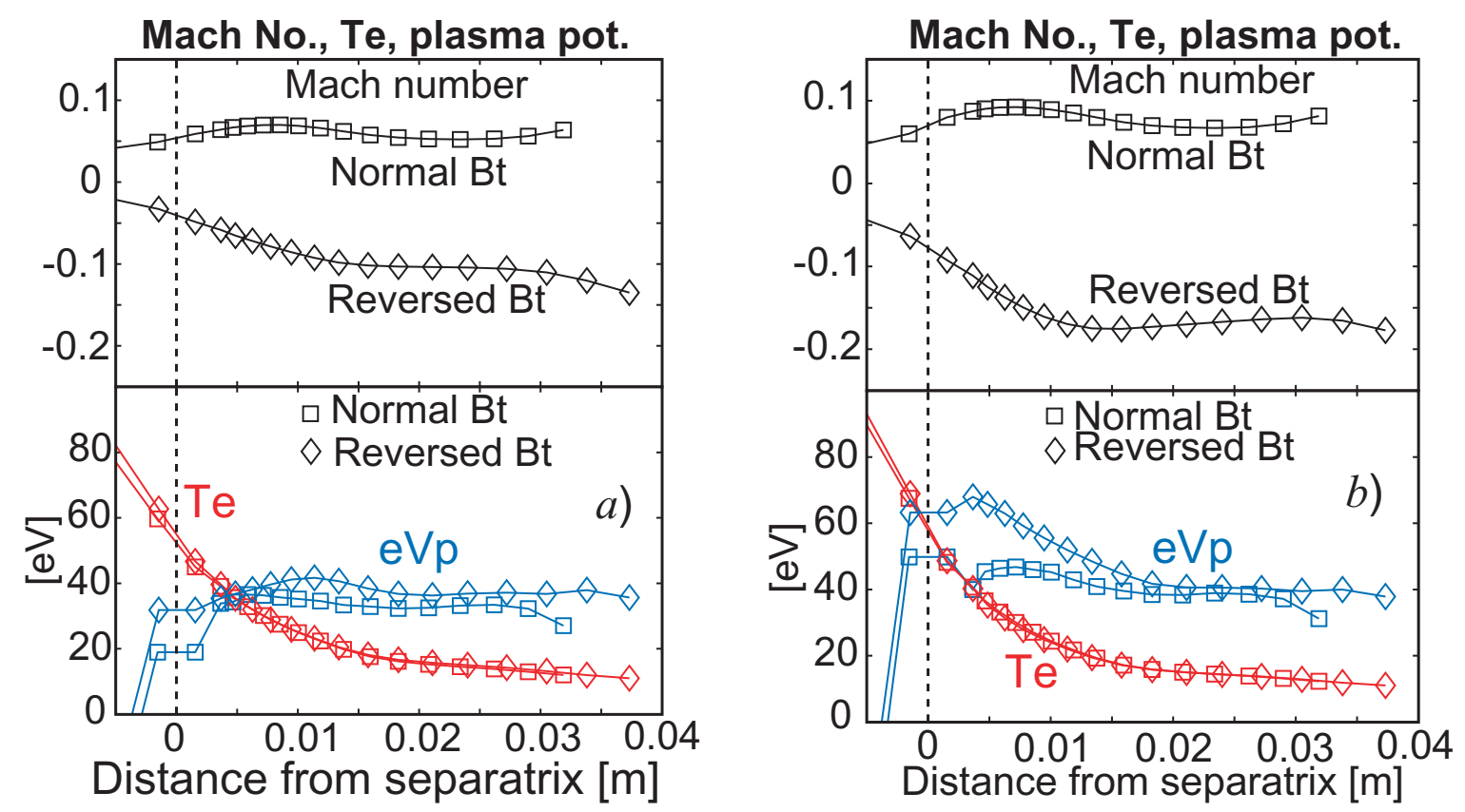

Fig. 1a,b 


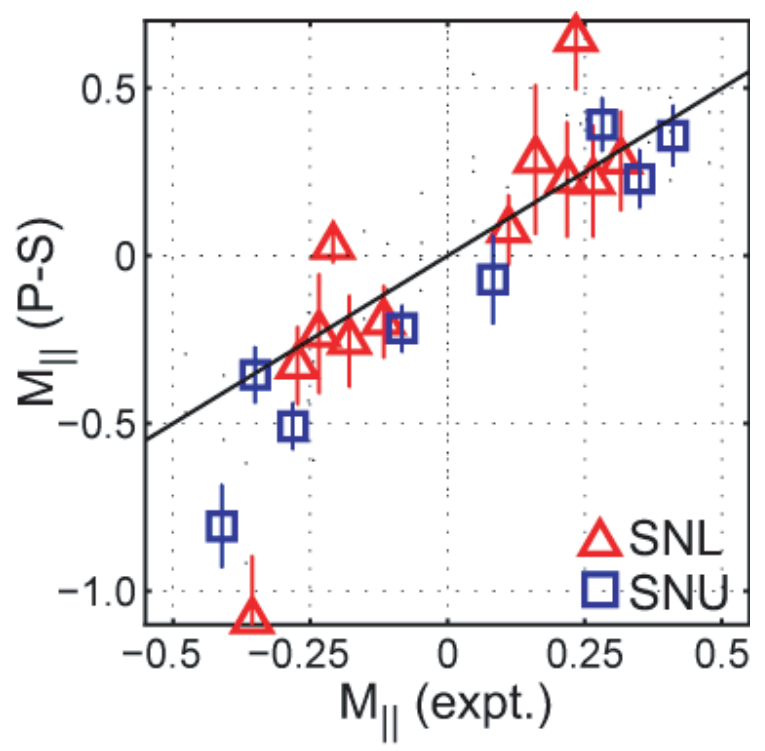

Fig. 2 


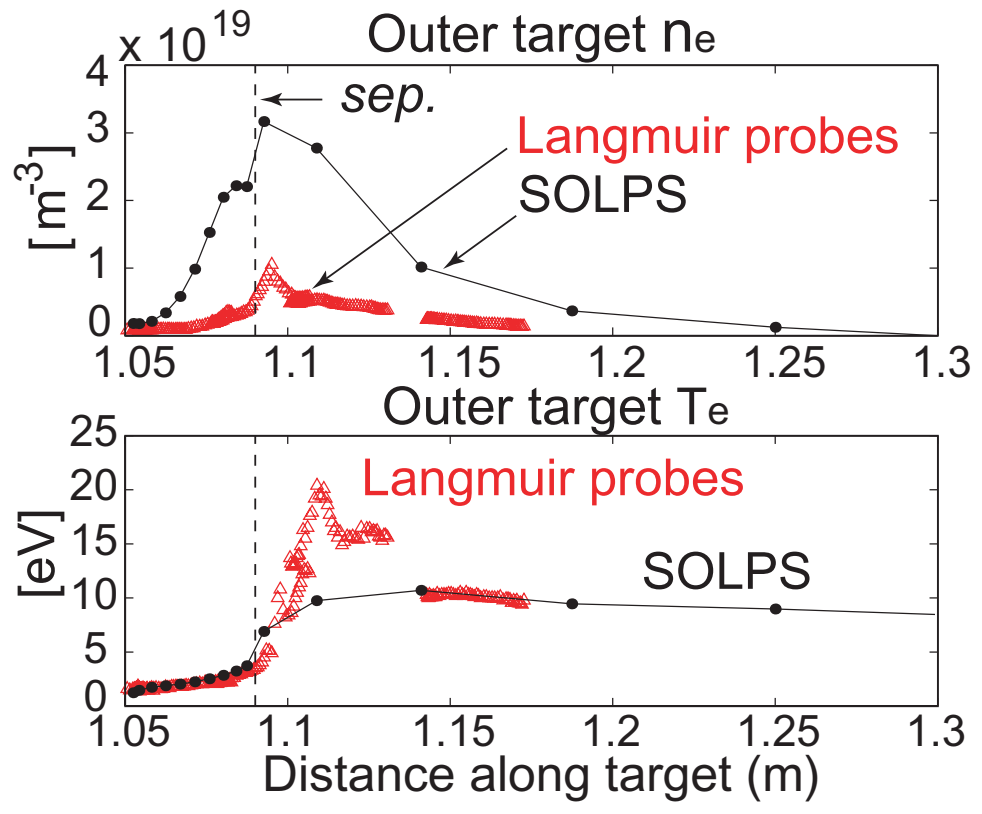

Fig. 3 


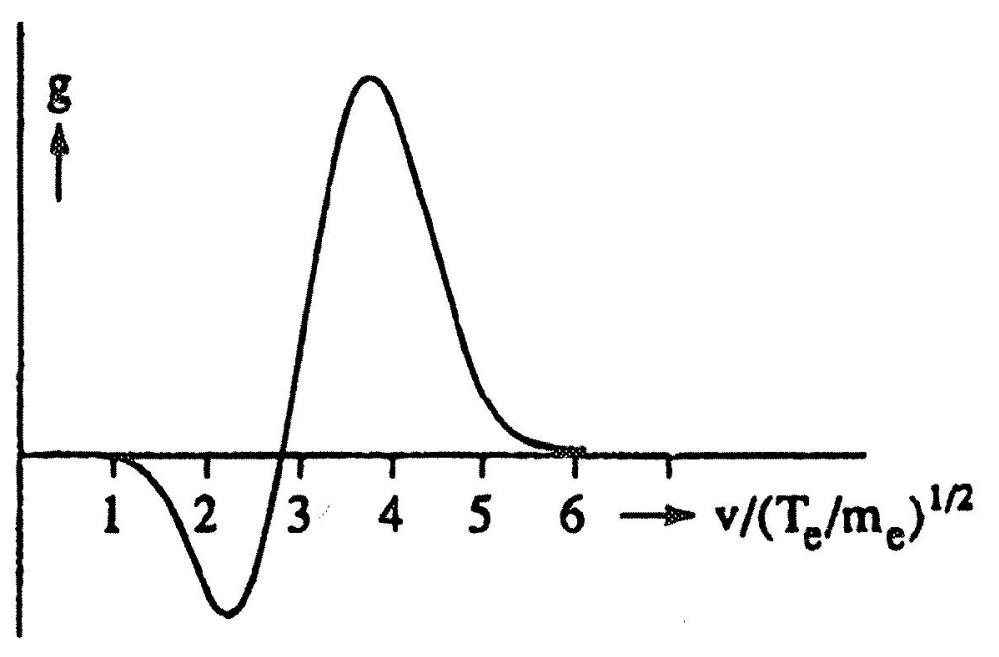

Fig. 4 


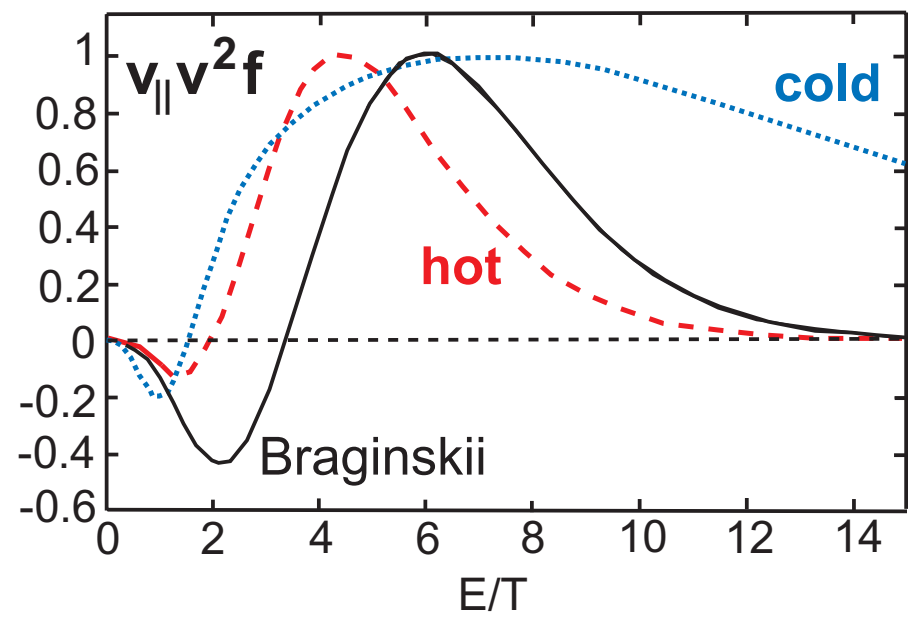

Fig. 5 